\title{
Síndrome dos ovários policísticos: aspectos clínicos e impactos na saúde da mulher
}

\author{
Polycystic ovary syndrome: clinical aspects and impacts on women's health \\ Síndrome de ovario poliquístico: aspectos clínicos e impactos en la salud de la mujer
}

Recebido: 26/01/2021 | Revisado: 02/02/2021 |Aceito: 06/02/2021 | Publicado: 14/02/2021

Igor dos Santos Cavalcante

ORCID: https://orcid.org/0000-0003-2907-9026 Universidade Federal do Delta do Parnaíba, Brasil E-mail: igorsc@live.com

Isabella Pires Gomes Mendes

ORCID: https://orcid.org/0000-0003-3486-7908 Universidade Federal do Delta do Parnaíba, Brasil E-mail: isabellapiresmendes@gmail.com

Martha Laura Leão dos Santos Silva

ORCID: https://orcid.org/0000-0003-2985-2461

Universidade Federal do Delta do Parnaíba, Brasil

E-mail: marthalauraleaodosssilva@gmail.com

Giovanna Stefanne Lópes Barbosa

ORCID: https://orcid.org/0000-0003-3387-1219

Universidade Federal do Delta do Parnaíba, Brasil E-mail: gistefanne@gmail.com

Luciana Eda Maximiano Hasegawa

ORCID: https://orcid.org/0000-0003-3864-0671 Instituto de Educação Superior do Vale do Parnaíba, Brasil E-mail: lu.emhasegawa@gmail.com

Ana Vitória Meireles Veiga

ORCID: https://orcid.org/0000-0003-2247-4824

Universidade Federal do Delta do Parnaíba, Brasil E-mail: vitmei@hotmail.com

Isabella Cabral Ferraz

ORCID: https://orcid.org/0000-0001-6699-0340

Universidade Federal do Delta do Parnaíba, Brasil E-mail: isabellacferraz17@gmail.com

Francisco Enson Souza Gomes

ORCID: https://orcid.org/0000-0002-4980-5817

Universidade Federal do Delta do Parnaíba, Brasil

E-mail: ensongomes1@gmail.com

Lúcia Maria de Sousa Aguiar dos Santos

ORCID: https://orcid.org/0000-0002-9316-2214 Centro Universitário UniFacid, Brasil E-mail:1msasantos@gmail.com

\begin{abstract}
Resumo
Objetivo: Descrever os aspectos clínicos da Síndrome dos Ovários Policísticos (SOP) e seu impacto na saúde da mulher. Metodologia: Revisão integrativa da literatura utilizando as bases de dados Scientific Eletronic Library Online (SciELO), PUBMED e Literatura Latino-Americana e do Caribe (LILACS). Resultados: A análise do quadro clínico da SOP foi dividida em cinco tópicos: influência da Resistência Insulínica (RI); relação com a obesidade; hiperandrogenismo e hirsutismo; Síndrome Metabólica (SM) e risco cardiovascular; e interferência na vida sexual. Nesse sentido, o estado hiperglicêmico gerado pela RI determinou o desenvolvimento de diabetes e acantose nigricante, enquanto que obesidade foi observada tanto como consequência da SOP, quanto como um fator agravante dos sintomas dessa síndrome. Ademais, a presença de SM foi influenciada pelo Índice de Massa Corpórea (IMC) e idade. Quanto à sexualidade, os estudos notaram a ocorrência de disfunção sexual, decorrente de alterações físicas e desordem hormonal, e de infertilidade, devido à não ovulação. Conclusão: Conclui-se que a SOP gera uma gama bastante heterogênea de sintomas, que se manifestam em mais de uma fase da vida da mulher e por isso é necessária uma abordagem holística à paciente. Além disso, os hábitos de vida saudáveis, como dieta balanceada e exercícios físicos, influenciam positivamente na melhora dos sintomas.
\end{abstract}

Palavras-chave: Síndrome do ovário policístico; Hiperandrogenismo; Resistência à insulina. 


\begin{abstract}
Objective: To describe the clinical aspects of Polycystic Ovary Syndrome (PCOS) and its impact on women's health. Methodology: Integrative literature review using the Scientific Eletronic Library Online (SciELO), PUBMED and Latin American and Caribbean Literature (LILACS) databases. Results: The analysis of the clinical picture of PCOS was divided into five topics: influence of Insulin Resistance (IR); relationship with obesity; hyperandrogenism and hirsutism; Metabolic Syndrome (MS) and cardiovascular risk; and interference with sexual life. In this sense, the hyperglycemic state generated by IR determined the development of diabetes and acanthosis nigricans, while obesity was observed both as a consequence of PCOS and as an aggravating factor in the symptoms of this syndrome. In addition, the presence of MS was influenced by the Body Mass Index (BMI) and age. As for sexuality, studies have noted the occurrence of sexual dysfunction, due to physical changes and hormonal disorder, and infertility, due to non-ovulation. Conclusion: We conclude that PCOS generates a very heterogeneous range of symptoms, which manifest themselves in more than one phase of a woman's life and, therefore, a holistic approach to the patient is necessary. In addition, healthy lifestyle habits, such as a balanced diet and exercise, positively influence the improvement of symptoms.
\end{abstract}

Keywords: Polycystic ovary syndrome; Hyperandrogenism; Insulin resistance.

\title{
Resumen
}

Objetivo: Describir los aspectos clínicos del Síndrome de Ovario Poliquístico (SOP) y su impacto en la salud de la mujer. Metodología: Revisión integrativa de la literatura utilizando las bases de datos Scientific Eletronic Library Online (SciELO), PUBMED y Literatura Latinoamericana y del Caribe (LILACS). Resultados: El análisis del cuadro clínico del SOP se dividió en cinco temas: influencia de la Resistencia a la Insulina (RI); relación con la obesidad; hiperandrogenismo e hirsutismo; Síndrome metabólico (EM) y riesgo cardiovascular; e interferencia con la vida sexual. En este sentido, el estado hiperglucémico generado por la RI determinó el desarrollo de diabetes y acantosis nigricans, mientras que la obesidad se observó tanto como consecuencia del SOP como agravante de los síntomas de este síndrome. Además, la presencia de EM fue influenciada por el índice de masa corporal (IMC) y la edad. En cuanto a la sexualidad, los estudios han señalado la aparición de disfunción sexual, debido a cambios físicos y desorden hormonal, e infertilidad, debido a la no ovulación. Conclusión: Concluimos que el SOP genera una gama de síntomas muy heterogénea, que se manifiesta en más de una fase de la vida de la mujer y, por tanto, es necesario un abordaje holístico de la paciente. Además, los hábitos de vida saludables, como una dieta equilibrada y el ejercicio, influyen positivamente en la mejora de los síntomas.

Palabras clave: Síndrome del ovario poliquístico, Hiperandrogenismo; Resistencia a la insulina.

\section{Introdução}

A Síndrome dos Ovários Policísticos (SOP) é uma das doenças endócrino-metabólicas mais comuns nas mulheres em idade reprodutiva, tendo sua prevalência de 9 a 18\%, variando de acordo com os critérios de diagnóstico empregados e a população estudada. Essa desordem endócrina é caracterizada por disfunção ovulatória e hiperandrogenismo, além de relacionar-se com complicações metabólicas e cardiovasculares. (Spritzer, 2014; Sidra, Tariq, Farrukh, \& Mohsin, 2019).

Em 2003, no Consenso de Rotterdam, foram definidos os critérios mais amplamente aceitos e abrangentes para diagnosticar a SOP. De acordo com Rotterdam, para o diagnóstico da síndrome é necessário que a paciente tenha pelo menos dois dos três critérios: hiperandrogenismo clínico e/ou laboratorial, oligo-amenorreia e critérios ultrassonográficos. Além disso, com base nesses critérios é possível observar a formação de quatro fenótipos de SOP: A: oligoovulação ou anovulação, hiperandrogenismo e ovários policísticos; B: oligoovulação ou anovulação e ovários policísticos; C: hiperandrogenismo e ovários policísticos; D: oligoovulação ou anovulação e hiperandrogenismo. (Febrasgo, 2018; Tavares \& Barros, 2019).

Segundo Spritzer (2014), a caracterização clínica dessa doença vai alterando-se ao longo da vida. No período póspuberal as mudanças fisiológicas do eixo hipotálamo - hipófise - ovário ainda não estão completamente consolidadas, resultando em irregularidades menstruais e ciclos anovulatórios, fato que dificulta o diagnóstico da SOP na adolescência. Desse modo, para a definição desta síndrome na pós-menarca é necessário que a adolescente tenha os três critérios de Rotterdam completos, não apenas dois, além da presença de hiperandrogenismo e anovulação após dois anos da menarca. Já no período de transição da menopausa, os distúrbios endócrinos se sobreporem aos ginecológicos. Nessa fase também há uma queda na produção androgênica, fato que torna difícil o diagnóstico. Em razão disso e das alterações clínicas marcantes do 
período, a caracterização dessa desordem é confirmada com base na história clínica da paciente, que normalmente ocorre antes da menopausa (Rehme, Pontes, Goldberg, Corrente \& Pontes, 2013; Bellver et al. 2017).

Essa patologia traz consequências não só fisiológicas, caracterizadas por distúrbios metabólicos importantes, mas também impactos psicossociais. A acne e o hirsutismo, por exemplo, são manifestações clínicas do hiperandrogenismo que afetam diretamente a autoestima das mulheres e consequentemente sua qualidade de vida. Além desses sintomas, que interferem no bem-estar social dessas pacientes, existem certas condições que as portadoras da SOP apresentam maior probabilidade de desenvolver, como doenças cardiovasculares, resistência insulínica, obesidade e até mesmo infertilidade. Diante disso, o diagnóstico precoce dessa doença é de fundamental importância para o rastreio e prevenção das complicações clínicas e psicossociais das mulheres acometidas. (Moura, Bagatin, Manela-Azulay, Costa, \& Sodré, 2011; Moreira, Sá, Costa, \& Azevedo, 2013)

Destarte, o presente estudo tem como objetivo descrever os aspectos clínicos da SOP, bem como os seus impactos na saúde da mulher.

\section{Metodologia}

Trata-se de um artigo de revisão, cuja abordagem adotada foi quantitativa, visando analisar e sintetizar o que a literatura traz sobre os aspectos clínicos relacionados à complexa síndrome dos ovários policísticos e quais os impactos que esse distúrbio provoca na saúde feminina. Essa pesquisa levou em consideração os fundamentos de uma revisão integrativa de literatura, os quais consistem em: (1) identificação do tema e seleção da questão norteadora; (2) definição de critérios de inclusão e exclusão de estudos; (3) investigação na literatura; (4) estabelecimento das informações a serem coletadas dos trabalhos selecionados; (5) análise dos estudos selecionados; (6) interpretação dos resultados e (7) síntese das informações.

A investigação dos artigos foi realizada utilizando os descritores: "Síndrome do Ovário Policístico", "Hiperandrogenismo" e "Resistência à insulina", obtidos nos Descritores em Ciências da Saúde (DECs), no Portal da Biblioteca Virtual em Saúde. Esses descritores foram combinados por operadores lógicos e buscados nas seguintes bases de dados: Scientific Eletronic Library Online (SciELO), PUBMED e Literatura Latino-Americana e do Caribe (LILACS).

Os critérios de inclusão foram: artigos científicos disponibilizados de forma integral, que estivessem enquadrados como pesquisas em seres humanos com ano de publicação entre 2010 e 2020. Os critérios de exclusão foram: materiais que não fossem artigos científicos e estudos que não estivessem de acordo com o tema selecionado.

Ao utilizar os três descritores associados na base LILACS, foram encontrados cinquenta e um artigos. Em seguida, a busca foi feita com os descritores em pares: com "síndrome do ovário policístico" e "hiperandrogenismo" foram encontrados cento e vinte e três artigos; o par "síndrome do ovário policístico" e "resistência à insulina" apresentou cento e trinta e oito artigos; já o par "resistência à insulina" e "hiperandrogenismo" teve vinte e nove artigos.

Na base SciELO, ao utilizar os três descritores em associação foram encontrados sete artigos; ao usar o par "síndrome do ovário policístico" e "hiperandrogenismo" foram encontrados dez artigos; utilizando o par "síndrome do ovário policístico" e "resistência à insulina" foram encontrados vinte e um artigos; o par "resistência à insulina" e "hiperandrogenismo" apresentou vinte e oito artigos

Na base PUBMED, ao utilizar os três descritores foram encontrados cento e oitenta e nove artigos; ao usar o par "síndrome do ovário policístico" e "hiperandrogenismo" foram encontrados trezentos e noventa e quatro artigos; utilizando o par "síndrome do ovário policístico" e "resistência à insulina" foram encontrados seiscentos e cinquenta e seis. Na busca com os descritores "hiperandrogenismo" e "resistência à insulina" resultou em duzentos e dez artigos. 
No total, foram encontrados mil oitocentos e cinquenta e seis artigos na busca inicial, dos quais foram descartados mil oitocentos e seis artigos por não estarem diretamente relacionados ao estudo. A seguir, sobraram cinquenta artigos para leitura, excluindo-se vinte e três por não adequação ao foco da discussão. Por conseguinte, foram selecionados vinte e sete artigos. Destes, foram excluídos sete devido a repetição. Por fim, sobraram vinte artigos para compor a pesquisa realizada. O fluxograma da Figura 1 detalha cada etapa da revisão de literatura realizada.

A pesquisa foi realizada em Outubro de 2020 e os artigos escolhidos foram submetidos a instrumento no software Microsoft Excel 2016 contendo as variáveis: título do artigo, ano de publicação, periódico e objetivo do estudo.

Figura 1 - Fluxograma detalhando cada etapa do processo de seleção de artigos para a revisão.

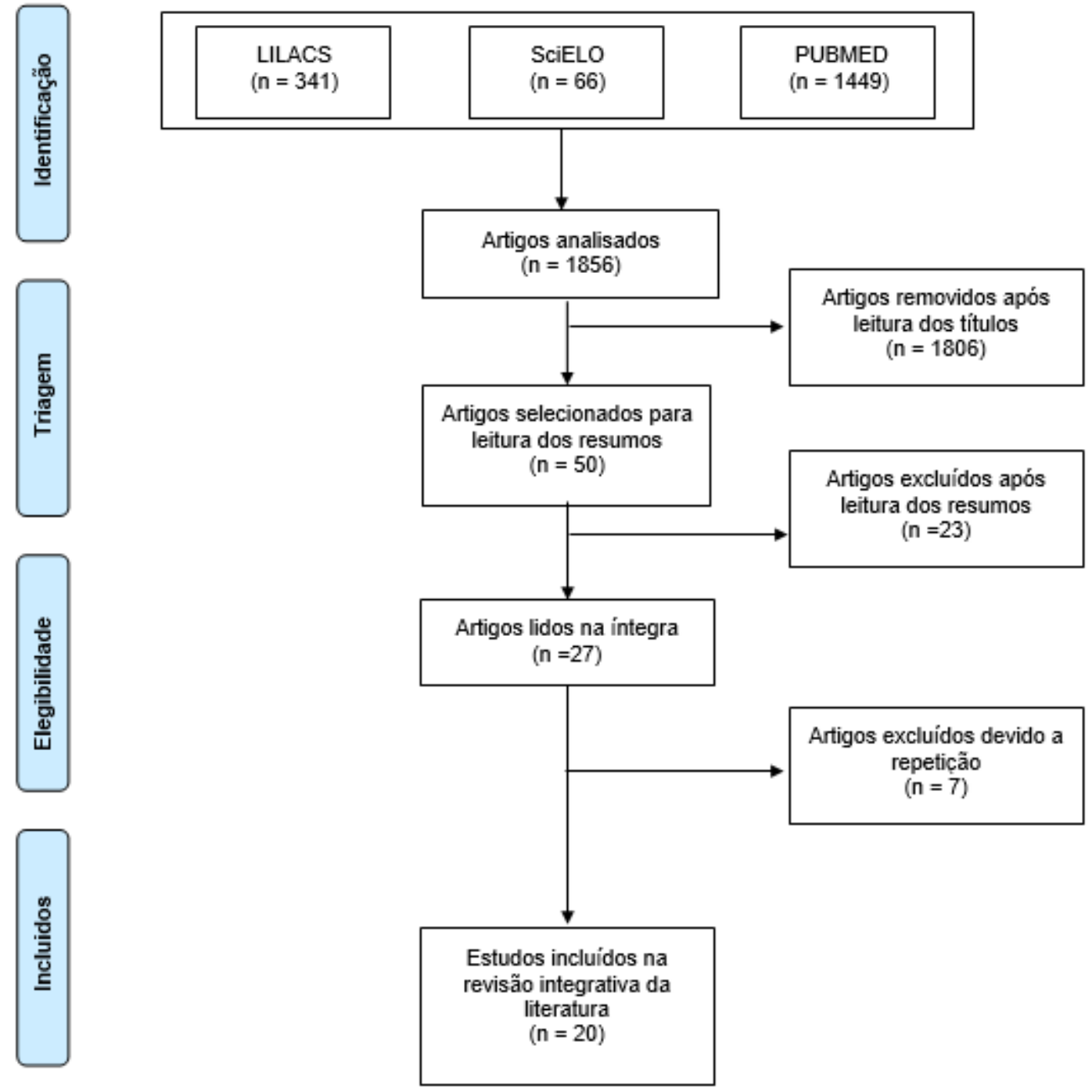

Fonte: Cavalcante et al. (2020)

\section{Resultados e Discussão}

Dentre os estudos encontrados, a amostra final foi constituída por artigos indexados nas três bases de dados descritas e que contemplaram todos os critérios de inclusão utilizados para validar os estudos no processo de busca bibliográfica. Os artigos incluídos foram de revistas diferentes e estão especificados de acordo com título e objetivo principal (Quadro 1). 
Quadro 1 - Artigos selecionados para a composição do estudo, abordando a revista, ano de publicação, objetivos e seus respectivos resultados principais.

\begin{tabular}{|c|c|c|c|c|}
\hline Obra & TÍTULO & ANO & PERIÓDICO & OBJETIVO \\
\hline 1 & $\begin{array}{l}\text { Índices de Obesidade Central e } \\
\text { Fatores de Risco Cardiovascular } \\
\text { na Síndrome dos Ovários } \\
\text { Policísticos }\end{array}$ & 2010 & $\begin{array}{l}\text { Arq } \\
\text { Cardiol }\end{array}$ & $\begin{array}{l}\text { Verificar a acurácia da circunferência da cintura, da relação } \\
\text { cintura-quadril, da relação cintura-estatura e do índice de } \\
\text { conicidade (índice C), no que se refere à detecção de fatores de } \\
\text { risco cardiovascular em mulheres com Síndrome dos ovários } \\
\text { policísticos }\end{array}$ \\
\hline 2 & $\begin{array}{l}\text { Participação dos androgênios } \\
\text { adrenais na síndrome dos } \\
\text { ovários policísticos }\end{array}$ & 2010 & $\begin{array}{l}\text { Rev Bras } \\
\text { Ginecol } \\
\text { Obstet. }\end{array}$ & $\begin{array}{l}\text { Reavaliar a função adrenal em pacientes com síndrome dos } \\
\text { ovários policísticos, após a introdução dos critérios de Roterdã. }\end{array}$ \\
\hline 3 & $\begin{array}{l}\text { Anormalidades metabólicas em } \\
\text { mulheres com síndrome dos } \\
\text { ovários policísticos: obesas e } \\
\text { não obesas }\end{array}$ & 2011 & $\begin{array}{l}\text { Rev Bras } \\
\text { Ginecol } \\
\text { Obstet. }\end{array}$ & $\begin{array}{l}\text { Comparar as características metabólicas de mulheres jovens do } \\
\text { sudeste brasileiro, obesas e não obesas com síndrome dos } \\
\text { ovários policísticos }\end{array}$ \\
\hline 4 & $\begin{array}{l}\text { Síndrome do ovário policístico: } \\
\text { abordagem dermatológica }\end{array}$ & 2011 & $\begin{array}{l}\text { An Bras } \\
\text { Dermatol, }\end{array}$ & $\begin{array}{l}\text { Avaliar as principais consequências dermatológicas da } \\
\text { síndrome dos ovários policísticos e como sua abordagem deve } \\
\text { ser feita }\end{array}$ \\
\hline 5 & $\begin{array}{l}\text { Mulheres com síndrome dos } \\
\text { ovários policísticos apresentam } \\
\text { maior frequência de síndrome } \\
\text { metabólica independentemente } \\
\text { do índice de massa corpóreo }\end{array}$ & 2012 & $\begin{array}{l}\text { Rev Bras } \\
\text { Ginecol } \\
\text { Obstet. }\end{array}$ & $\begin{array}{l}\text { Avaliar a prevalência de síndrome metabólica e dos seus } \\
\text { critérios definidores em mulheres com síndrome dos ovários } \\
\text { policísticos, estratificadas de acordo com o índice de massa } \\
\text { corpóreo e comparadas com controles ovulatórias }\end{array}$ \\
\hline 6 & $\begin{array}{l}\text { Resistência à insulina em } \\
\text { mulheres com síndrome dos } \\
\text { ovários policísticos: relação com } \\
\text { as variáveis antropométricas e } \\
\text { bioquímicas }\end{array}$ & 2012 & $\begin{array}{l}\text { Rev Bras } \\
\text { Ginecol } \\
\text { Obstet. }\end{array}$ & $\begin{array}{l}\text { Analisar a prevalência de resistência à insulina de acordo com } \\
\text { diferentes medidas antropométricas e bioquímicas em } \\
\text { mulheres com síndrome dos ovários policísticos }\end{array}$ \\
\hline 7 & $\begin{array}{l}\text { Polycystic ovary syndrome is } \\
\text { associated with higher left } \\
\text { ventricular mass index: the } \\
\text { CARDIA women's study }\end{array}$ & 2012 & $\begin{array}{l}\text { J Clin } \\
\text { Endocrinol } \\
\text { Metab }\end{array}$ & $\begin{array}{l}\text { O objetivo do estudo foi determinar se mulheres jovens com } \\
\text { síndrome dos ovários policísticos (SOP) apresentam evidências } \\
\text { de alterações estruturais precoces nos parâmetros } \\
\text { ecocardiográficos como medida de risco cardiovascular. }\end{array}$ \\
\hline
\end{tabular}




\begin{tabular}{|c|c|c|c|c|}
\hline 8 & $\begin{array}{l}\text { Arterial hypertension and } \\
\text { metabolic profile in patients } \\
\text { with polycystic ovary syndrome }\end{array}$ & 2013 & $\begin{array}{l}\text { Rev Bras } \\
\text { Ginecol } \\
\text { Obstet. }\end{array}$ & $\begin{array}{l}\text { Avaliar os parâmetros relacionados com a pressão arterial e o } \\
\text { perfil metabólico em portadoras de SOP }\end{array}$ \\
\hline 9 & $\begin{array}{l}\text { Contribuição do } \\
\text { hiperandrogenismo } \\
\text { para o desenvolvimento de } \\
\text { síndrome } \\
\text { metabólica em mulheres obesas } \\
\text { com } \\
\text { síndrome dos ovários } \\
\text { policísticos }\end{array}$ & 2013 & $\begin{array}{l}\text { Rev } \\
\text { Ginecol } \\
\text { Obstet. }\end{array}$ & $\begin{array}{l}\text { Avaliar a contribuição do hiperandrogenismo para o } \\
\text { desenvolvimento da síndrome metabólica em } \\
\text { mulheres obesas com ou sem Síndrome dos Ovários } \\
\text { Policísticos }\end{array}$ \\
\hline 10 & $\begin{array}{l}\text { Manifestações clínicas, } \\
\text { bioquímicas, ultrassonográficas } \\
\text { e metabólicas da síndrome dos } \\
\text { ovários policísticos em } \\
\text { adolescentes }\end{array}$ & 2013 & $\begin{array}{l}\text { Rev Bras } \\
\text { Ginecol } \\
\text { Obstet. }\end{array}$ & $\begin{array}{l}\text { Avaliar os parâmetros clínicos, ultrassonográficos, } \\
\text { bioquímicos e as alterações metabólicas em adolescentes com } \\
\text { síndrome dos ovários policísticos }\end{array}$ \\
\hline 11 & $\begin{array}{l}\text { Qualidade de vida e aspectos } \\
\text { psicossociais da síndrome dos } \\
\text { ovários policísticos: um estudo } \\
\text { quali-quantitativo }\end{array}$ & 2013 & $\begin{array}{l}\text { Rev } \\
\text { Ginecol } \\
\text { Obstet. }\end{array}$ & $\begin{array}{l}\text { Avaliar a qualidade de vida das mulheres com síndrome dos } \\
\text { ovários policísticos e compreender a experiência vivida por } \\
\text { essas mulheres diante dos sintomas que apresentam }\end{array}$ \\
\hline 12 & $\begin{array}{l}\text { Polycystic ovary syndrome: } \\
\text { reviewing } \\
\text { diagnosis and management } \\
\text { of metabolic disturbances }\end{array}$ & 2014 & $\begin{array}{l}\text { Arq Bras } \\
\text { Endocrinol } \\
\text { Metab. }\end{array}$ & $\begin{array}{l}\text { Revisar os principais aspectos relacionados com o diagnóstico } \\
\text { e manejo dos distúrbios metabólicos presentes na síndrome dos } \\
\text { ovários policísticos }\end{array}$ \\
\hline 13 & $\begin{array}{l}\text { Acantose nigricante: inter- } \\
\text { relações metabólicas inerentes à } \\
\text { síndrome dos ovários } \\
\text { policísticos }\end{array}$ & 2014 & $\begin{array}{l}\text { Rev Bras } \\
\text { Ginecol } \\
\text { Obstet. }\end{array}$ & $\begin{array}{l}\text { Avaliar a contribuição do hiperandrogenismo para o } \\
\text { desenvolvimento da síndrome metabólica em mulheres obesas } \\
\text { com ou sem Síndrome dos Ovários Policísticos }\end{array}$ \\
\hline 14 & $\begin{array}{l}\text { Síndrome dos ovários } \\
\text { policísticos: implicações da } \\
\text { disfunção } \\
\text { metabólica }\end{array}$ & 2014 & $\begin{array}{l}\text { Rev. Col. } \\
\text { Bras. Cir. }\end{array}$ & $\begin{array}{l}\text { Estabelecer a prevalência da síndrome metabólica e as } \\
\text { respectivas inter-relações clínicas no contexto da síndrome } \\
\text { dos ovários policísticos }\end{array}$ \\
\hline
\end{tabular}




\begin{tabular}{|c|c|c|c|c|}
\hline 15 & $\begin{array}{l}\text { Ferriman-Gallwey Score } \\
\text { correlates with obesity and } \\
\text { insulin levels } \\
\text { in Polycystic Ovary Syndrome - } \\
\text { an observational study }\end{array}$ & 2015 & $\begin{array}{l}\text { Rev Soc Bras } \\
\text { Clin Med. }\end{array}$ & $\begin{array}{l}\text { O presente estudo teve como objetivo avaliar a correlação entre } \\
\text { hiperandrogenismo clínico, avaliado pela escala de Ferriman- } \\
\text { Gallwey modificada e parâmetros metabólicos em mulheres } \\
\text { com a Síndrome dos Ovários Policísticos }\end{array}$ \\
\hline 16 & $\begin{array}{l}\text { Sexual Dysfunction in Patients } \\
\text { with Polycystic Ovary } \\
\text { Syndrome in Malaysia }\end{array}$ & 2016 & $\begin{array}{l}\text { Asian Pac J } \\
\text { Cancer Prev. }\end{array}$ & $\begin{array}{l}\text { o objetivo foi avaliar a frequência e os fatores predisponentes } \\
\text { da disfunção sexual em pacientes com SOP. }\end{array}$ \\
\hline 17 & $\begin{array}{l}\text { Polycystic ovary syndrome } \\
\text { throughout a woman's life }\end{array}$ & 2017 & $\begin{array}{l}\text { J Assist } \\
\text { Reprod Genet }\end{array}$ & $\begin{array}{l}\text { O objetivo da presente revisão é descrever o } \\
\text { consequências da síndrome desde o início da vida reprodutiva } \\
\text { até o seu fim de acordo com a literatura médica atual }\end{array}$ \\
\hline 18 & $\begin{array}{l}\text { The Prevalence of Metabolic } \\
\text { Syndrome in the Different } \\
\text { Phenotypes of Polycystic } \\
\text { Ovarian Syndrome }\end{array}$ & 2019 & $\begin{array}{l}\text { Rev Bras } \\
\text { Ginecol } \\
\text { Obstet }\end{array}$ & $\begin{array}{l}\text { Avaliar a prevalência da síndrome metabólica nos fenótipos } \\
\text { da síndrome do ovário policístico }\end{array}$ \\
\hline 19 & $\begin{array}{l}\text { Psychiatric disorders in women } \\
\text { with polycystic ovary syndrome }\end{array}$ & 2019 & Psychiatr Pol & $\begin{array}{l}\text { Este artigo pretende fornecer uma visão geral da literatura } \\
\text { sobre } \\
\text { Distúrbios mentais em mulheres com síndrome dos ovários } \\
\text { policísticos. }\end{array}$ \\
\hline 20 & $\begin{array}{l}\text { Evaluation of clinical } \\
\text { manifestations, health risks, and } \\
\text { quality of life among women } \\
\text { with polycystic ovary syndrome }\end{array}$ & 2019 & PLoS One & $\begin{array}{l}\text { Este estudo teve como objetivo avaliar as manifestações } \\
\text { clínicas e os riscos à saúde associados à síndrome dos ovários } \\
\text { policísticos (SOP) e seu impacto na qualidade de vida (QV) no } \\
\text { Paquistão. }\end{array}$ \\
\hline
\end{tabular}

Fonte: Cavalcante et al (2020).

\section{Resultados e Discussão}

\section{Influência da resistência insulínica}

De acordo com Pontes et al. (2012), define-se resistência à insulina (RI) como um estado metabólico no qual ocorre desequilíbrio na homeostase dos níveis glicêmicos, levando a uma falha no metabolismo da glicose.

O estado de hiperinsulinemia resultante da RI é responsável pela elevação da produção androgênica no organismo feminino, devido à ação desse hormônio estimulando diretamente as células da teca ovariana. Além disso, a insulina também atua na diminuição da produção da proteína carregadora de androgênios (Steroid Hormone Binding Globuline - SHBG) no fígado. Assim, com base nesses processos, ocorre uma elevação da concentração da testosterona livre, o principal androgênio, na circulação sanguínea (Spritzer, 2014).

As evidências científicas demonstram que há uma forte correlação entre a presença do mecanismo de RI e o desenvolvimento da SOP (Moura et al., 2011). Segundo pesquisa realizada por Tavares e Barros (2019), a qual avaliou 163 
pacientes, a prevalência encontrada de RI em mulheres com SOP foi de 39,6\%. Rehme, Pontes, Goldberg et al. (2013) encontrou associação de RI em $62,8 \%$ da população adolescente na amostra investigada, o que corrobora com os dados apresentados pela literatura.

Em outro estudo, realizado por Pontes et al. (2012), identificou-se que há uma variação na frequência da RI dependendo do método de rastreamento utilizado: o método com maior detecção para RI foi o Índice de Sensibilidade à Insulina (ISI), com resultado positivo para 56,4\% das pacientes avaliadas, seguido da insulinemia de jejum, com 49,6\%, Quantitative Insulin Sensitivity Check Index (QUICKI) e Homeostasis Model Assessment For Insulin Resistance (HOMA-IR), com $46,3 \%$, e, por fim, o parâmetro de relação glicemia e insulinemia de jejum apresentou resultado de $42 \%$.

Destaca-se que o método padrão-ouro para rastreamento de RI é o teste de clamp euglicêmico, no entanto, por causa de seu elevado custo e prolongado tempo para realização, o uso desse exame é bastante limitado na prática médica, especialmente nos serviços públicos (Romano et al. 2011).

Quanto às manifestações clínicas presentes na SOP decorrentes da RI no organismo, tem-se a diabetes como principal consequência da hiperglicemia, como mostrou a pesquisa de Sidra et al. (2019), na qual a diabetes estava presente em 60,9\% das 440 pacientes estudadas. Em gestantes com SOP, a principal complicação relacionada é a Diabetes Mellitus Gestacional (DMG), com risco estimado para ocorrência maior de 2 a 3 vezes em comparação com gestantes sem a síndrome (Bellver et al. 2017).

Outra manifestação importante consiste na Acantose Nigricante (AN), que corresponde a um marcador cutâneo da condição de elevada taxa glicêmica no organismo e possui grande associação com a RI (Moura et al. 2011). Caracterizada por uma mancha aveludada e acastanhada na pele, a AN possui relevante prevalência em pacientes com SOP, como descreve Ávila, Bruno et al. (2014) em seu estudo, que identificou presença em 53\% das investigadas. Por isso, de acordo com esse autor, a AN deve ter inspeção rotineira, pois a sua presença qualifica um fenótipo de gravidade na SOP e possui relação intrínseca com disfunções metabólicas.

\section{Relação com a obesidade}

A obesidade é uma doença caracterizada pelo acúmulo de gordura corporal de forma excessiva, prejudicando a saúde do indivíduo (Wanderley \& Ferreira, 2010). Segundo a Organização Mundial da Saúde [OMS] (1998), uma pessoa é considerada obesa quando seu Índice de Massa Corpórea (IMC) é maior ou igual a $30 \mathrm{~kg} / \mathrm{m}^{2}$, o qual é determinado da seguinte forma: divide-se o peso de uma pessoa, em quilogramas, pelo quadrado da sua altura, medida em metros. Frequentemente, a obesidade faz parte do quadro clínico da SOP, estando presente em 30\% a 70\% das mulheres com essa síndrome (Sales, Sóter, Cândido, Reis, Sousa, Fernandes, Ferreira \& Gomes, 2015; Melo, Macedo, Romano, Ferriani \& Navarro, 2012).

A correlação entre SOP e obesidade é complexa, mas alguns estudos sugerem que existe uma relação entre a adiposidade e a severidade das manifestações clínicas das mulheres com essa síndrome, ou seja, a obesidade pode agravar o quadro clínico da paciente, exacerbando distúrbios metabólicos e reprodutivos que estão associados comumente à SOP (Spritzer, 2014; Sales et al. 2015; Tavares \& Barros, 2019). Segundo uma análise de Rehme, Pontes, Corrente, Franco Júnior e Pontes (2013), a obesidade por si só interfere no ciclo menstrual das mulheres, alterando seu padrão, já que, neste estudo, todas as mulheres obesas que apresentavam SOP manifestaram anovulação, e mesmo nas mulheres obesas que não tem SOP, a anovulação estava presente. O mesmo estudo ainda mostrou que todas as mulheres obesas com SOP tinham hiperandrogenismo, em detrimento do grupo de mulheres obesas sem a SOP, no qual nenhuma apresentou essa manifestação característica da síndrome (Rehme, Pontes, Corrente et al., 2013). 
De acordo com o estudo de Melo et al. (2012), feito com mulheres que apresentam SOP na região sudeste do Brasil, o grupo de mulheres obesas com SOP teve uma frequência de Síndrome Metabólica (SM) de 67,9\%, frequência seis vezes maior quando comparada ao grupo controle de mulheres obesas sem a SOP, das quais 10,5\% apresentavam a SM, o que demonstra essa associação entre obesidade e o agravamento do perfil metabólico dessas pacientes. Este mesmo estudo ainda traz que 80,9\% das mulheres obesas com SOP apresentaram hiperandrogenismo clínico, demonstrando uma maior frequência neste grupo em detrimento de mulheres com o IMC abaixo de $30 \mathrm{~kg} / \mathrm{m}^{2}$. Outrossim, Romano e colaboradores trazem em seu estudo que a frequência de RI e SM em obesas com SOP é de 66,7\% e 63\%, respectivamente, enquanto nas pacientes com SOP não obesas, essas porcentagens são, respectivamente, 24,7\% e 16,4\% (Romano et al., 2011).

Na tentativa de explicar essa interferência da obesidade na severidade clínica da SOP, vários estudos analisaram a fisiopatologia do tecido adiposo. Segundo Rehme, Pontes, Corrente et al., (2013), o tecido adiposo é metabolicamente ativo e, na obesidade em vigência de SOP, há uma predominância da obesidade hipertrófica, em que há um aumento no tamanho dos adipócitos, em detrimento da obesidade hiperplásica, caracterizada pelo aumento no número dessas células. Essa predominância da obesidade hipertrófica em mulheres com SOP sugere que a capacidade lipolítica dos adipócitos esteja desequilibrada e, assim, esse prejuízo no metabolismo de lipídios nessas mulheres poderia exacerbar os distúrbios metabólicos como resistência à insulina e síndrome metabólica. (Oliveira, Redorat, Ziehe, Mansur \& Conceição, 2012; Rehme, Pontes, Corrente et al., 2013)

Outro estudo que tenta explicar essa relação entre a obesidade e a SOP, é o de Tavares e Barros (2019), segundo o qual há dois aspectos importantes na fisiopatologia da síndrome e da obesidade que formam uma espécie de retroalimentação: hiperandrogenismo e hiperinsulinemia. De acordo com os autores, o hiperandrogenismo da SOP aumenta a expressão dos genes envolvidos com a lipogênese, o que predispõe o acúmulo de gordura. Assim, é favorecido o desenvolvimento ou agravo de obesidade, situação já estabelecida de agravo de RI. Além disso, na SOP, a RI com a hiperinsulinemia compensatória estimula os ovários e as adrenais a produzir andrógenos, agravando o quadro de hiperandrogenismo.

Em pesquisa de Sales et al. (2015), ainda pode-se observar a relação da obesidade com o desequilíbrio de hormônios sexuais em mulheres. Nesse estudo, fala-se que os níveis de SHBG tendem a diminuir na medida em que a gordura corporal aumenta, o que causa um aumento da fração livre de andrógenos no sangue, podendo agravar um quadro de hiperandrogenismo.

Outras evidências científicas que apoiam essa relação íntima da obesidade com a severidade dos sinais e sintomas da SOP é o fato de que uma das linhas de tratamento de SOP consiste na alteração no estilo de vida, principalmente no que diz respeito à regularidade na prática de atividade físicas e a dietas balanceadas, sendo que a partir do início do tratamento, o objetivo deve ser reduzir pelo menos 5\% a 10\% do peso da paciente (Bellver et al. 2017; Moura et al. 2011).

Segundo estudo de Sales et al. (2015), podem haver melhorias significativas no quadro da paciente, resultando em regularidade no ciclo menstrual, atenuando características hiperandrogênicas e até mesmo melhorando o quadro de infertilidade, em consequência à redução de peso mínima. De acordo com Moura et al. (2011), estes efeitos na severidade do quadro clínico são relacionados à redução nos níveis de insulina e, consequentemente, melhora da resistência insulínica. Assim, essa linha de tratamento pode contribuir para melhorar a qualidade de vida das pacientes obesas com SOP, além de prevenir outras comorbidades ou complicações dessas doenças.

Entretanto, vale ressaltar que, apesar das evidências mostrarem que a obesidade pode agravar os quadros clínicos e exacerbar os riscos de distúrbios associados à SOP, a prevalência desses distúrbios metabólicos é elevada também nas pacientes não obesas mas que apresentam a SOP, sugerindo que, independente do IMC da paciente, a síndrome por si só já favorece o desenvolvimento de comorbidades metabólicas. (Romano et al. 2011). 


\section{Hiperandrogenismo e hirsutismo}

Os achados fundamentais para o diagnóstico da SOP durante a adolescência se evidenciam por critérios delimitados, como o ciclo menstrual irregular representado pela oligomenorreia e/ou amenorreia por, pelo menos, dois anos após a menarca, a ultrassonografia apresentando ovários policísticos e volume ovariano maior ou igual a $10 \mathrm{~cm}^{3}$ e sinais clínicos e/ou bioquímicos de hiperandrogenismo (Rehme, Pontes, Goldberg et al., 2013).

Nesse contexto, na SOP, a produção exacerbada de androgênios se relaciona com a atuação das adrenais e dos ovários. O aumento dos androgênios ovarianos é o mais evidente e provoca especialmente a elevação da testosterona, advinda do hiperestímulo do LH, fortalecida pela ampliação intrínseca da secreção destes androgênios nas células da teca ou pela ação da insulina. Já o aumento dos androgênios adrenais acontece em 20 a $60 \%$ das pacientes com a patologia, caracterizando-se por

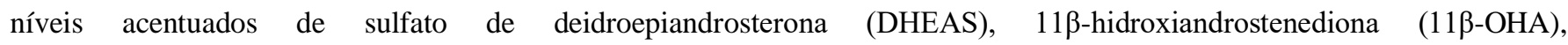
deidroepiandrosterona (DHEA), androstenediol e androstenediona. Com efeito, ainda não estão inteiramente esclarecidos os mecanismos do hiperandrogenismo adrenal na SOP, todavia, a maior proposição tem sido o catabolismo do cortisol e/ou a resposta aumentada dos androgênios adrenais a níveis normais do hormônio adrenocorticotrófico (ACTH) (Gil Junior, Rezende, Carmo, Duarte, Medeiros \& Medeiros, 2010).

Nas mulheres, o hiperandrogenismo acarreta manifestações clínicas de diferentes intensidades, como acne, alopecia, seborréia, irregularidades menstruais, puberdade precoce, distúrbios psicológicos, síndrome metabólica, virilização e hirsutismo. O hirsutismo constitui-se como um parâmetro clínico primário e se caracteriza com o crescimento exacerbado de pelos terminais faciais ou corporais, em decorrência da elevação de andrógeno ou ampliação da sensibilidade do folículo capilar para níveis normais de androgênio. Perante essa circunstância, utiliza-se a pontuação de Ferriman-Gallwey (F-G) para definir a gravidade do hirsutismo, analisando a expansão do crescimento dos pelos perante nove sítios anatômicos (Sales et al., 2015).

Outro aspecto relevante sobre o hirsutismo está na determinação de que as mulheres com hirsutismo possuem uma amplificação da atividade da enzima 5a-redutase nos folículos pilosos, essa atividade é estimulada tanto pelo hiperandrogenismo, quanto pelo fator de crescimento insulina-like e a própria insulina. Além disso, a testosterona e dihidrotestosterona (DHT) estabelecem as alterações no pelo, transformando o pêlo vellus em terminal, com maior espessura e pigmentação, nas áreas andrógeno-sensíveis como a face, o pescoço, o tórax e a região pubiana (Moura et al., 2011).

Diante disso, deve-se ressaltar que a extensão e a intensidade das manifestações clínicas abordadas dependem de fatores variados e, portanto, não existe uma relação restrita entre a intensidade das condições clínicas e alterações nos níveis bioquímicos. Assim, recomenda-se a avaliação clínica do hiperandrogenismo para discernir as pacientes hiperandrogênicas que não possuem alterações nos níveis de andrógenos em exames laboratoriais (Sales et al., 2015).

\section{Síndrome Metabólica e Risco Cardiovascular}

De acordo com a I Diretriz Brasileira de Diagnóstico e Tratamento da Síndrome Metabólica (2005), a SM é uma condição complexa, normalmente relacionada à deposição central de gordura e à resistência à insulina e que resulta no aumento do risco cardiovascular. Sua relação com a SOP é alvo de diferentes pesquisas nos últimos anos, na busca por se estabelecer a prevalência e principais indicadores clínicos para a suspeição e diagnóstico das pacientes que somam essas duas síndromes (Oliveira et al., 2013; Rehme, Pontes, Corrente et al., 2013; Spritzer, 2014).

Trabalhos com pacientes adultas com SOP do nordeste e sudeste brasileiro apontaram prevalência da associação SOP/SM entre 33,6 e 59\% (Ávila, Bruno et al., 2014; Melo et al., 2012; Tavares \& Barros, 2019). 
Ao considerar a faixa etária na porcentagem de diagnósticos do binômio SOP/SM, observa-se que cerca de 14\% das adolescentes com SOP também apresentam SM (Rehme, Pontes, Goldberg et al., 2013). Esse menor número de adolescentes com SM, no contexto da SOP, parece estar relacionada às mudanças no quadro clínico de pacientes com SOP ao longo dos anos, de forma que adolescentes com SOP apresentam, em maior frequência, sinais relacionados ao hiperandrogenismo e à oligo/anovulação, enquanto que naquelas de idade avançada, além dessas duas categorias, apresentam também sintomas metabólicos, como alguns sintomas vasomotores, sobrepeso ou obesidade, resistência à insulina e SM (Bellver et al., 2017).

Outro parâmetro de grande relevância no estudo da associação entre SOP e SM é o IMC. E, ao adicionar essa variável no estudo da prevalência da SOP associada à SM, observam-se conflitos entre os estudos selecionados. Enquanto Melo et al. (2004) concluem em seu estudo que um maior número de diagnósticos de SM é encontrado em mulheres com SOP, independentemente do IMC, quando comparadas a controles sem SOP - com diferença estatisticamente significativa diminuindo a importância da obesidade no desencadeamento da SM; Romano et al. (2011) observaram que 63,3\% das mulheres obesas com SOP de seu estudo apresentavam SM versus 16,7\% em não-obesas com SOP ( $<<0,01)$, associação também observada por Rehme, Pontes, Corrente et al. (2013) ( $\mathrm{p}=0,01$ ), o que indicaria maior influência da obesidade no desenvolvimento da SM em pacientes com SOP.

O diagnóstico da SM baseia-se fundamentalmente em cinco parâmetros, dos quais a presença de pelo menos três determina o diagnóstico: pressão arterial (PA), HDL-colesterol, triglicerídeos, glicemia de jejum e circunferência abdominal (CA). E, embora existam diferentes diretrizes que ditam os critérios diagnósticos da $\mathrm{SM}$, todas parecem concordar nos pontos de corte referentes à PA, com valores normais abaixo de $130 \mathrm{mmHg}$ para PA sistólica e de $85 \mathrm{mmHg}$ para PA diastólica; HDLcolesterol, acima de $50 \mathrm{mg} / \mathrm{dL}$; triglicerídeos, abaixo de $150 \mathrm{mg} / \mathrm{dL}$ e glicemia de jejum, abaixo de $100 \mathrm{mg} / \mathrm{dL}$ (Alberti et al., 2009; Grundy et al., 2005).

Contudo, os valores de referência relacionados à $\mathrm{CA}$, indicativos da obesidade centrípeta dos pacientes, variam conforme a referência utilizada, podendo ser, em mulheres sul-americanas, maior ou igual a $80 \mathrm{~cm}$, como preconizado pela International Diabetes Federation e utilizado por Ávila, Borges et al. (2014), Rehme, Pontes, Goldberg et al. (2013) e Tavares \& Barros (2019); ou ainda, maior ou igual a $88 \mathrm{~cm}$, de acordo com o proposto pela American Heart Association e empregado por Melo et al. (2012), Pontes et al. (2013) e Romano et al. (2011) (Alberti et al., 2009).

Estudos realizados por Ávila, Borges et al. (2014) e também por Costa, Soares, Lemos, Maranhão e Azevedo (2010) apontaram um valor médio de circunferência abdominal de mulheres adultas com SOP de cerca de $90 \mathrm{~cm}$. Estudos utilizando o ponto de corte para CA de $80 \mathrm{~cm}$ encontraram $65,8 \%$ e $63,6 \%$ de prevalência de pacientes com SOP com CA aumentada para adultas e adolescentes, respectivamente (Tavares e Barros, 2019; Rehme, Pontes, Goldberg et al., 2013).

Ao adicionar o componente IMC à estratificação dessas mulheres, pode-se observar médias que vão de $80,5 \mathrm{~cm}$ para pacientes com IMC $<25$ a 110,3 cm para aquelas com IMC $>30$ e índices de até $100 \%$ de circunferência aumentada, mesmo considerando o limite de $88 \mathrm{~cm}$ para esse parâmetro, como observado nos trabalhos de Rehme, Pontes, Goldberg et al. (2013) e Melo et al. (2012). Dessa forma reafirma-se a importância da obesidade como componente do binômio SOP/SM.

Em pesquisas realizadas por Costa et al. (2010) e Tavares e Barros (2019), valores de PAS e PAD encontram-se alterados em cerca de $20 \%$ das pacientes com SOP. Valores elevados de PA em mulheres com SOP parecem estar positivamente associados ao IMC. Melo et al. (2012) observaram que 59\% das 105 mulheres obesas com SOP de seu estudo tinham PA>130/85 mmHg, semelhante ao observado por Tavares e Barros (2019) (53,3\%). E, embora adultas e idosas estejam mais predispostas a essas alterações pressóricas, adolescentes portadoras de SOP também podem apresentar PA elevada. Rehme, Pontes, Goldberg et al. (2013) encontraram uma prevalência de 9,1\% de PA alterada em adolescentes com SOP. 
Considerando os artigos selecionados, o nível sérico de HDL-colesterol por sua vez, é a variável com maiores índices de alterações. Trabalhos realizados por Costa et al. (2010), Melo et al. (2012) e Tavares e Barros (2019) encontraram respectivamente, 54,1\%, 73\% e 76,5\% de pacientes com SOP e valores séricos de HDL-colesterol abaixo de $50 \mathrm{mg} / \mathrm{dL}$. Sendo ainda superior em trabalho que analisou apenas pacientes com SOP obesas (IMC >30), no qual 85\% contavam com a alteração (Rehme, Pontes, Corrente et al., 2013).

A respeito dos níveis séricos de triglicerídeos em mulheres portadoras de SOP, Costa et al. (2010), ao analisar 186 mulheres com SOP e estratifica-las conforme o IMC, obtiveram como médias de triglicerídeos: 92,3 mg/dL para o grupo de $\mathrm{IMC}<25,113,5 \mathrm{mg} / \mathrm{dL}$ para o de IMC entre 25 e 30 e $150,0 \mathrm{mg} / \mathrm{dL}$ para o grupo de IMC $>30$. Isso indica mais uma vez que a obesidade está relacionada aos parâmetros para definição da SM no grupo de mulheres com SOP.

Sobre a glicemia de jejum, intrinsecamente relacionada à resistência à insulina, aqui já discutida, observaram-se menores porcentagens de valores alterados nos trabalhos selecionados, quando comparadas aos demais parâmetros de diagnóstico da SM. Tavares e Barros (2019) observaram em estudo com 163 mulheres diagnosticadas com SOP que 7,2\% das mulheres estavam com glicemia de jejum acima de $100 \mathrm{mg} / \mathrm{dL}$. Contudo, como para outras variáveis da SM, a glicemia de jejum alterada tende a ser mais frequente em mulheres obesas. Melo et al. (2012) encontraram uma prevalência de 24,5\% de glicemia de jejum alterada em mulheres obesas portadoras de SOP, ao passo que Rehme, Pontes, Corrente et al. (2013) encontraram uma porcentagem de 30,3\% para pacientes com o mesmo perfil.

Além desses parâmetros já utilizados para o diagnóstico da SM, outros indicadores mostram associação com fatores de risco cardiovascular em pacientes com SOP. Costa et al. (2010) avaliaram indicadores antropométricos e sua relação com fatores de risco cardiovascular, sendo a Relação Cintura-Estatura o índice que apresentou melhores correlações positivas com PA, TG e glicemia pós-TOTG, e importante correlação negativa com os níveis de HDL-colesterol. Isso é de grande relevância, uma vez que a simplicidade da mensuração da estatura e da circunferência abdominal podem antecipar a suspeita clínica para o risco cardiovascular, antes mesmo da realização de exames laboratoriais que - a depender da realidade da paciente - poderiam demorar muito a serem realizados.

\section{Interferência na vida reprodutiva}

Segundo Dashti et al. (2016), a disfunção sexual é definida como um problema que ocorre em alguma das fases da resposta sexual. Essa alteração em mulheres com síndrome dos ovários policísticos (SOP) pode decorrer das alterações físicas que, frequentemente, diminuem a autoestima, como o hirsutismo, obesidade e acne, e também da desordem nos hormônios sexuais.

As manifestações físicas decorrentes do hiperandrogenismo interferem na atividade sexual. Sinais como o hirsutismo podem fazer com que a mulher sinta perda de identidade e consequente redução da libido. Dessa forma, muitas pacientes são prejudicadas no âmbito conjugal e adquirem problemas com o parceiro. Além disso, essa situação gera estresse psicológico na mulher e reduz a sensação de bem estar (Moreira et al., 2013).

Alterações hormonais também estão presentes em pacientes com SOP e interferem sobremaneira na saúde reprodutiva. Nesses casos, a proporção entre o Hormônio Luteinizante (LH) e o Hormônio Folículo Estimulante (FSH) está alterada pelo excesso de LH. Consequentemente, ocorre um aumento da quantidade de andrógenos que gera excesso nos níveis de estrógenos, ciclo menstrual irregular e anovulação, importante fator para a infertilidade (Moura et al., 2011).

Uma pesquisa, realizada por Dashti et al. (2016) avaliou 16 mulheres diagnosticadas com SOP para avaliar a disfunção sexual. Os itens do questionário foram divididos nos seguintes domínios: desejo, excitação, lubrificação, orgasmo, satisfação e dor. Dentre esses, a disfunção sexual na área da excitação foi a maior, presente em 98,3\% das mulheres e na área 
do orgasmo foi a menor, presente em 18,8\%. Outrossim, os resultados do questionário demonstraram que as alterações na sexualidade em mulheres com SOP está, em grande parte, associada a níveis mais baixos de educação e ao hirsutismo.

Além da redução na atividade sexual, a infertilidade também está presente em muitos casos. Resultante dos distúrbios relacionados à ovulação, a dificuldade de engravidar torna-se comum em pacientes com SOP. A anovulação manifesta-se clinicamente e deve ser identificada por meio de características do ciclo menstrual sendo frequente a amenorreia ou oligomenorreia. Nesse processo, é importante que as possibilidades para o tratamento da infertilidade sejam apresentadas para a mulher. Quanto à medicação a ser utilizada, enquanto diretrizes mais antigas recomendam a utilização de citrato de clomifeno, as mais recentes apontam o letrozol como melhor opção por possuir maior eficácia (Sidra et al. 2019).

Outro fator de relevância nesse quadro clínico é a obesidade. Se a mulher for obesa e infértil, o tratamento de primeira escolha acontece por meio da mudança no estilo de vida. No caso do tratamento não apresentar resultado, ou se a obesidade não estiver presente, a utilização de medicamentos é indicada (Bellver et al. 2017).

As interferências na sexualidade de mulheres com SOP necessitam de reconhecimento e intervenção precoce, para que haja o tratamento adequado. Nesses casos, depressão e ansiedade podem estar presentes e a atuação do profissional de saúde para encaminhamento psicológico é de fundamental importância para melhoria da qualidade de vida das mulheres (Moreira et al., 2013).

\section{Conclusão}

Por conseguinte, conclui-se que a SOP é uma condição clínica que acomete as mulheres em mais de uma fase da vida, manifestando-se com uma ampla variedade de sintomas e impactando negativamente na qualidade de vida das pacientes. Fazse necessário, assim, o acompanhamento holístico das mulheres portadoras dessa síndrome, evidenciando tanto seu aspecto biológico quanto social. Torna-se indispensável, para a melhora dos sintomas apresentados, além de um tratamento farmacológico adequado, uma mudança no estilo de vida, associada com práticas dietéticas saudáveis e rotina regular de exercícios físicos.

\section{Referências}

Alberti, K. G. M. M., Eckel, R. H., Grundy, S. M., Zimmet, P. Z., Cleeman, J. I., Donato, K. A., Fruchart, J. C., James, W. P. T., Loria, C. M., \& Smith Jr, S. C. (2009). Harmonizing the metabolic syndrome: a joint interim statement of the international diabetes federation task force on epidemiology and prevention; national heart, lung, and blood institute; American heart association; world heart federation; international atherosclerosis society; and international association for the study of obesity. Circulation, 120(16), 1640-45. https://doi.org/10.1161/circulationaha.109.192644.

Ávila M. A. P., Borges L. P., Paez M. S., Bruno R. V., Nardi A. E., Pessôa A. C. M., Palmeira E. S. (2014). Acantose nigricante: inter-relações metabólicas inerentes à síndrome dos ovários policísticos. Rev Bras Ginecol Obstet. 36(9):410-5. http://dx.doi.org/10.1590/SO100-720320140005078.

Ávila M. A. P., Bruno R. V., Barbosa F. C., Andrade F. C., Silva A. C. O., Nardi A. E. (2014). Síndrome dos ovários policísticos: implicações da disfunção metabólica. Rev. Col. Bras. Cir. 41(2): 106-11. https://doi.org/10.1590/S0100-69912014000200006.

Bellver J., Rodríguez-Tabernero L., Robles A., Muñoz E., Martínez F., Landeras J., García-Velasco J., Fontes J., Álvarez M., Álvarez C., Acevedo B. (2018). Polycystic ovary syndrome throughout a woman's life. J Assist Reprod Genet. 35:25-39. https://dx.doi.org/10.1007\%2Fs10815-017-1047-7.

Costa E. C., Soares E. M. M., Lemos T. M. A. M., Maranhão T. M. O., Azevedo G. D. (2010). Índices de obesidade central e fatores de risco cardiovascular na síndrome dos ovários policísticos. Arq. Bras. Cardiol. 94 (5):633-38. http://dx.doi.org/10.1590/S0066-782X2010005000029.

Dashti S., Lattif L. A., Hamid H. A., Sani S. M., Akhtari-Zavare M., Bakar A. S. A., Sabri N. A. I. B., Ismail M., Esfehani A. J. (2016). Sexual Dysfunction in Patients with Polycystic Ovary Syndrome in Malaysia. Asian Pac J Cancer Prev, 17 (8): 3747-51. http://journal.waocp.org/article_33048_88 d7908ddc7bc886baa5077ae8cce06a.pdf.

Federação Brasileira das Associações de Ginecologia e Obstetrícia. (2018). Síndrome dos ovários policísticos. https://www.febrasgo.org.br/media/k2/attachments/18Z-ZSndromeZdosZovriosZpolicsticos.pdf. 
Gil Junior A. B., Rezende A. P. R., Carmo A. V., Duarte E. I., Medeiros M. M. W. Y., Medeiros S. F. (2010). Participação dos androgênios adrenais na síndrome dos ovários policísticos. Rev Bras Ginecol Obstet. 32(11):541-8. https://doi.org/10.1590/\$0100-72032010001100005.

Grundy, S. M., Cleeman, J. I., Daniels, S. R., Donato, K. A., Eckel, R. H., Franklin, B. A., Gordon, D. J., Krauss, R. M., Savage, P. J., Smith, S. C., Jr, Spertus, J. A., Costa, F., American Heart Association, \& National Heart, Lung, and Blood Institute (2005). Diagnosis and management of the metabolic syndrome: an American Heart Association/National Heart, Lung, and Blood Institute Scientific Statement. Circulation, 112(17), 2735-52. https://doi.org/10.1161/CIRCULATIONAHA.105.169404.

Melo A. S., Macedo C. S. V., Romano L. G. M., Ferriani R. A., Navarro P. A. A. S. (2012). Mulheres com síndrome dos ovários policísticos apresentam maior frequência de síndrome metabólica independentemente do índice de massa corpóreo. Rev Bras Ginecol Obstet. 34(1):4-10. 2020 de https://doi.org/10.1590/S0100-72032012000100002.

Moreira S. N., Sa J. C., Costa E. C., Azevedo G. D. (2013). Qualidade de vida e aspectos psicossociais da síndrome dos ovários policísticos: um estudo qualiquantitativo. Rev Bras Ginecol Obstet. 35(11):503-10. https://doi.org/10.1590/S0100-72032013001100005.

Moura H. H. G., Costa D. L. M., Bagatin E., Sodré C. T., Azulay M. M. (2011). Síndrome do ovário policístico: abordagem dermatológica. An Bras Dermatol. 86(1):111-9. http://dx.doi.org/10.1590/S0365-05962011000100015.

Oliveira R. S. M., Redorat R. G., Ziehe G. H., Mansur V. A., Conceição F. L. (2013). Arterial hypertension and metabolic profile in patients with polycystic ovary syndrome. Rev Bras Ginecol Obstet. 35(1):21-6. https://doi.org/10.1590/S0100-72032013000100005.

Pereira, A. S., et al. (2018). Metodologia da pesquisa científica. UFSM. https://repositorio.ufsm.br/bitstream/handle /1/15824/Lic_Computacao_MetodologiaPesquisa-Cientifica.pdf? sequence $=1$

Pontes A. G. (2012). Resistência à insulina em mulheres com síndrome dos ovários policísticos: relação com as variáveis antropométricas e bioquímicas. Rev Bras Ginecol Obstet. 34(2):74-9. https://doi.org/10.1590/S0100-72032012000200006.

Rehme M. F. B., Pontes A. G., Corrente J. E., Franco Jr J. G., Pontes A. (2013). Contribuição do hiperandrogenismo para o desenvolvimento de síndrome metabólica em mulheres obesas com síndrome dos ovários policísticos. Rev Bras Ginecol Obstet. 35(12):562-8. http://dx.doi.org/10.1590/S010072032013001200006.

Rehme M. F., Pontes A. G., Goldberg T. B., Corrente J. E., Pontes A. (2013). Manifestações clínicas, bioquímicas, ultrassonog ráficas e metabólicas da síndrome dos ovários policísticos em adolescentes. Rev Bras Ginecol Obstet. 35(6):249-54. https://doi.org/10.1590/S0100-72032013000600003.

Rodriguez-Paris D., Remlinger-Molenda A., Kurzawa R., Głowińska A., Spaczyński R., Rybakowski F., Pawelczyk L., Banaszewska B. (2019). Psychiatric disorders in women with polycystic ovary syndrome. Psychiatr. Pol. 53(4): 955-66. https://doi.org/10.12740/PP/OnlineFirst/93105.

Romano L. G. M., Bedoschi G., Melo A. S., Albuquerque F. O., Silva A. C. J. S. R., Ferriani R. A., Navarro P. A. A. S. (2011). Anormalidades metabólicas em mulheres com síndrome dos ovários policísticos: obesas e não obesas. Rev Bras Ginecol Obstet. 33(6):310-6. https://doi.org/10.1590/S010072032011000600008 .

Sales M. F., Sóter M. O., Cândido A. L., Reis F. M., Sousa M. O., Fernandes A. P., Ferreira C. N., Gomes K. B. (2015). Ferriman-Gallwey Score correlates with obesity and insulin levels in Polycystic Ovary Syndrome - an observational study. Rev Soc Bras Clin Med. 13(2):107-10. http://files.bvs.br/upload/S/1679-1010/2015/v13n2/a4737.pdf.

Sidra S., Tariq M. H., Farrukh M. J., Mohsin M. (2019). Evaluation of clinical manifestations, health risks, and quality of life among women with polycystic ovary syndrome. PLoS ONE 14(10): e0223329. https://doi.org/10.1371/journal.pone.0223329.

Sociedade Brasileira de Cardiologia (2005). I Diretriz Brasileira de Diagnóstico e Tratamento da Síndrome Metabólica. Arquivos Brasileiros de Cardiologia. 84(Suppl.1), 3-28. https://doi.org/10.1590/ S0066-782X2005000700001.

Spritzer P. M. (2014). Polycystic ovary syndrome: reviewing diagnosis and management of metabolic disturbances. Arq Bras Endocrinol Metab. 58(2):182-7. http://dx.doi.org/10.1590/0004-2730000003051.

Tavares A., \& Barros R. C. R. (2019). The prevalence of metabolic syndrome in the different phenotypes of polycystic ovarian syndrome. Rev Bras Ginecol Obstet. 41:37-43. http://dx.doi.org/10.1055/s-0038-1676568.

Wanderley, E. N. \& Ferreira, V. A. (2010). Obesidade: uma perspectiva plural. Ciência \& Saúde Coletiva. 15: 185-94. 2020 de https://doi.org/10.1590/S141381232010000100024

World Health Organization (1998). Obesity: Preventing and managing the global epidemic. Report of a WHO Consultation on Obesity. https://apps.who.int/iris/bitstream/handle/10665/63854/WHO_NUT_NCD_98.1_\%28p1-158\%29.pdf?sequence=1\&isAllowed=y. 